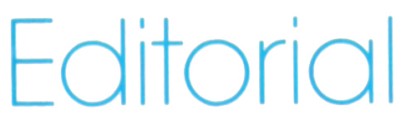

Harold C. Standiford, MD

\title{
Methicillin-resistant Staphylococcus aureus Infections: It's Time to Get Tough
}

In 1982 Boyce and Causey reported in Infection Control the results of a survey conducted to determine the magnitude of methicillin-resistant Staphylococcus aureus (MRSA) infections in the United States. From responses to that questionnaire, it was apparent that the number of hospitals reporting MRSA dramatically increased from 1975 to 1980 , that larger hospitals were encountering the problem more frequently than smaller ones, and that it was more common in university and federal hospitals than community, community teaching, and municipal hospitals. ${ }^{1}$

In this issue of Infection Control, Preheim and colleagues have determined that the problem has not abated, at least in the Federal Veterans Administration system. Since 1980, the hospitals identifying the problem have continued to increase. This increase is geographically widespread and involves hospitals of all sizes. ${ }^{2}$

Federal hospitals in general have a high incidence of nosocomial infections compared with community hospitals. ${ }^{3}$ The reasons for this are not absolutely known but are probably multiple. VA hospitals provide care for primarily indigent patients, a group that may delay therapy longer and have more severe underlying conditions. The patients are frequently older and stay somewhat longer than those in other hospitals. Fixed hospital budgets often produce short-

From Division of Infection Diseases, Department of Medicine, Baltimore Veterans Administration Medical Center and the University of Maryland School of Medicine, Baltimore, Maryland.

Address reprint requests to Harold C. Standiford, $M D$, Chief, Section of Infectious Diseases, Baltimore Veterans Administration Medical Center, 3900 Loch Raven Boulevard, Baltimore, MD 21218. ages in personnel, a problem aggravated by the scarcity of nurses in many areas of the country. Perhaps more important, there is an increased number of physicians and students per patient in the medical school-affiliated VA hospitals, as in university hospitals, each increasing the chances of spread of resistant pathogens from one patient to another. So it is in this type of setting, whether a university, VA, county, or community hospital, that the spread of resistant organisms such as MRSA can quickly get out of hand. It is in this type of setting that we must be particularly careful to prevent this from occurring.

The presence of these organisms in the hospital is costly. Vancomycin, the only parenteral antibiotic for therapy of severe infections from this organism, costs our hospital $\$ 900$ for a 2-week regimen. Likewise, the cost of isolation is not insignificant. But more important than cost are the morbidity and mortality caused by this organism in the hospital and its potential spread into chronic care facilities, nursing homes, and the community. As Preheim and colleagues have indicated, $41 \%$ of Veterans Administration Medical Centers reported sending known MRSA colonized patients to nursing homes-sometimes, I suspect, without forewarning the receiving institution that the patient has the resistant pathogen. Indeed, community identification has now been reported in Boston and New York, and has been noted in Baltimore and, I am sure, other communities as well. ${ }^{4,5,6}$ That the organism is in the community population is not only possible but appears to be a fact. It is our responsibility to prevent this from occurring or minimize it as much as we possibly can.

The following are recommendations we have found helpful in maintaining control of an MRSA outbreak in a 291- 
bed university-affiliated Veterans Administration hospital. Some of the recommendations were derived from research at the University of Virginia as well as at the Baltimore VA Medical Center. ${ }^{7,8}$ I present them here in the hope that they may help other institutions respond to what can be a difficult and frustrating experience.

1. Accepting the Problem. Often during the initial confrontation of the MRSA epidemic a defeatist attitude is adopted and arms are thrown up in submission because so many of the patients in the hospital are colonized or infected. The tendency is to accept the resistant pathogen as part of the normal hospital flora or take the attitude of one resident who stated, "If it weren't this resistant organism it would be another." At this point, a commitment must be made and led by the infection control personnel. Citing references indicating successful handling of outbreaks by others and the impact of the alternative on the hospital budget may help. Although the cost of outbreaks may not be the prime consideration of the infection control personnel, identifying monetary impact is one method to obtain the attention of the institution's administration, an essential element for a successful program. Because of the affinity of this organism for wounds, infected patients often reside among surgical patients. Therefore, it is essential to gain surgical support early in the control efforts.

2. Defining the Epidemiology. When the organism is identified, meticulous records should be maintained with attention to when the patient became infected, site of infection, place in the hospital at the time of pathogen acquisition, dates and places of intrahospital transfers, and association with other infected or colonized patients and, most important, physicians and nursing teams caring for the patient. This information should be analyzed so that cultures for hospital carriers, observation of wound care techniques, and education can be directed appropriately.

3. Identifying the Reservoir. The presence of MRSA is frequently discovered by the clinical microbiology laboratory during routine cultures from infected patients. This method identifies about one third of the patients within the hospital who are colonized or infected. ${ }^{7}$ Another one third can be identified by prospective microbiologic surveillance of all open wounds and tracheostomy sites, regardless of the presence of infection. It has been shown that culturing patients' nares, and probably rectal cultures, are unnecessary since these sites are seldom positive unless a wound is also positive. ${ }^{8}$ Medical care personnel need to be cultured only if they appear to be highly associated with the epidemic; that is, if the outbreak is localized on one ward or restricted to patients taken care of by one team of physicians. The associated personnel should be identified and nares and hands cultured.

Prospective microbiologic cultures are costly. However, methods to reduce costs of these prospective cultures are available, and their cost can easily be justified by the increased expense necessary for vancomycin during epidemic situations. ${ }^{8}$

The final one third of the total hospital reservoir can be identified by culturing readmissions known to be infected or colonized by MRSA during their previous admission. A list of these patients should be updated monthly and can be maintained at the admission desk and at the nursing stations in each patient care area. This list can easily be placed on the hospital computer if the equipment is available. These colonized or infected individuals are thereby identified immediately on readmission and placed on appropriate isolation until culture results are negative. By these three methods, the entire hospital reservoir can be identified. In large hospitals the process of identifying the reservoir can be focused in the high-risk areas if the outbreak is localized.

4. Isolation and Cohortation for Infected and Colonized Patients. By far, the most common mode of spreading this organism is transient carriage on the hands of health care workers to susceptible patients who are in proximity of other infected or colonized patients. It has been clearly demonstrated that hands of personnel are positive for MRSA immediately after dressing infected wounds. It also has been shown that MRSA experimentally inoculated on the hands of health care workers persists for more than 3 hours and, furthermore, that the organism can be eliminated by simple handwashing with soap and water. ${ }^{7}$ Unfortunately, it has also been shown that health care workers, particularly physicians, was their hands between patient examinations about $30 \%$ of the time. ${ }^{9}$ Thus, in addition to handwashing, some barriers to the spread of infection are essential.

As noted by Preheim et al, the type of isolation used by hospitals varies, although about half the hospitals surveyed employed contact isolation for both infected and colonized patients. For the epidemic situation, we choose strict isolation to present a high level of importance and prevent potential spread after touching contaminated objects in the room, but this is most likely overkill. The important point is that there is strict adherence to the isolation procedures, regardless of which type is chosen. Equally important is that disposable gloves be available and used throughout the hospital for care and dressing of all open surgical wounds, diabetic foot ulcers, and decubital and tracheostomy sitesregardless of whether they are known to be infected or colonized with MRSA or other resistant organisms. I have noted some improvement in this area, particularly by house staff and students, and would like to ascribe it to an effective education program. However, in more pessimistic moods, I ascribe it more to the health care worker's fear of catching AIDS rather than a conscious effort to prevent the spread of resistant bacteria.

In the midst of hospital outbreaks involving relatively large numbers of patients, it is necessary to group patients who are infected or colonized together to make it more efficient for medical care in the isolation setting. At times, open and crowded intensive care units have to be closed and temporary units containing uninfected patients opened. Temporary nurses may have to be hired to deal with the patient load, adding further to the cost of the outbreak. Cohortation of infected patients can be extremely helpful in larger epidemics to help manage the patients and minimize the costs.

5. Eradication of the Reservoir. Treatment of MRSA infections with vancomycin generally improves the infection but frequently may not eradicate the organism. Additionally, many other patients are colonized or have low-grade wound infections. We have elected to treat most of these patients with oral antibiotics to eradicate the organism. Although this may diminish the opportunity for subsequent systemic infection with the organism, antibiotic treatment is primarily 
aimed at reducing the hospital reservoir and decreasing the potential for community spread when the patient is discharged. Although data in this area are meager, Ward and colleagues have used rifampin $300 \mathrm{mg}$ orally twice daily along with topical bacitracin ointment. ${ }^{10}$ This appeared effective for nasal colonization, but resistance to rifampin frequently occurred with wound infections. The combination of rifampin with trimethoprim-sulfamethoxazole twice daily for 5 days with hexachlorophene baths on the initial two days was effective in 13 of 16 patients with wounds and tracheostomy site colonization and in all 10 individuals in whom colonization occurred in the anterior nares only. Other investigators have used regular whole body antiseptic washing, but the data for this are very limited."

6. Education. This is perhaps the most obvious yet most difficult aspect of any MRSA containment program. The education must touch all components of the medical care team including nurses, physicians, medical administration, and building management. By far the most difficult (and most important) personnel to reach are the "frontline" house staff and students. At the Baltimore VA Medical Center residents, interns, and medical students rotate from two different parent institutions, each group on different and usually monthly rotations. To reach all of them in their orientation period is a feat of major proportions. The job is compounded, in my opinion, by relatively little formal training in the medical school curriculum for infection control problems, procedures, and techniques. Relative to educating these groups, the permanent, nonrotating medical staff, nurses, medical administration, and building management personnel are easy to teach.

Total eradication of MRSA from the hospital is generally impossible. Numerous studies have identified that the organism is widespread in dust and inanimate objects such as patient charts, blood pressure cuffs, and even on tourniquets used for blood drawing, which may, in part, account for this difficulty. ${ }^{8,12}$ At the Baltimore VA Medical Center, small outbreaks occur almost yearly. For that reason we have elected to continue our prospective cultures of wounds and tracheostomy sites and maintain our admission MRSA identification lists so that new patients and readmissions can be quickly identified and the organism appropriately contained. It is clear that successful containment of this organism requires acceptance of the organism as a problem, and vigorous control measures by the entire medical team. Nevertheless, the additional efforts for its control are important to our patients, the hospital, and perhaps most important to the community into which our patients and their pathogens are discharged.

\section{REFERENCES}

1. Boyce JM, Causey WA: Increasing occurnence of methicillin-resistant Staphylococcus aureus in the United States. Infect Comtrol 1982; 3:377-383.

2. Preheim LC, Rimland D, Bittner MJ: Methicillin-resistant Staphylococcus aureus in Veterans Administration Medical Centers. Infect Control 1987; 8:191-194.

3. Haley $\mathrm{RW}$, Culver $\mathrm{DH}$, White $\mathbf{J W}$, et al: The nationwide nosocomial infection rate: A new need for vital statistics. Am J Epidemiol 1985; 121:159-167.

4. Craven DE, Rixinger AI, Goularte TA, et al: Methicillin-resistant Staphylococcus aureus bacteremia linked to intravenous drug abusers using a "shooting gallery". A $m$ J Med 1986; 80:770-776.

5. Saravolatz LD, Markowitz N, Arking L, et al: Methicillin-resistant Staphylococcus aureus: Epidemiologic observations during a community-acquired outbreak. Ann Intern Med 1982; 96:11-16.

6. Storch GA, Radcliff ]L, Meyer PL, et al: Methicillin-resistant Staphylococcus aureus in a nursing home. Infect Control 1987; 8:24-29.

7. Thompson RL, Cabezudo I, Wenzel RP: Epidemiology of nosocomial infections caused by methicillin-resistant Staphylococcus aureus. Ann Intern Med 1982; 97:309-317.

8. Walsh TJ, Vlahov D, Hansen SL, et al: Prospective microbiologic surveillance in control of nosocomial methicillin-resistant Staphylococcus aureus. Infect Control 1987: 8:7-14.

9. Albert BK, Condie $\mathbf{F}$ : Hand-washing patterns in medical intensive care units. $N$ Engl J Med 1981; 304:1465-1469.

10. Ward TГ, Winn RE, Hartstein AI, et al: Observations relating to an inter-hospital outbreak of methicillin-resistant Staphylococcus aureus: Role of antimicrobial therapy in infection control. Infect Control 1981; 2:453-459.

11. Bartzokas CA, Paton JH, Gibson MF, et al: Control and eradication of methicillinresistant Staphylococcus aureus on a surgical unit. $N$ Engl J Med 1984; 311:1422-1425.

12. Berman DS, Schaffler S, Simberkoff MS, et al: Tourniquets and nosocomial methicillin-resistant Staphylococcus aureus infections. N Engl J Med 1986; 315:514. 


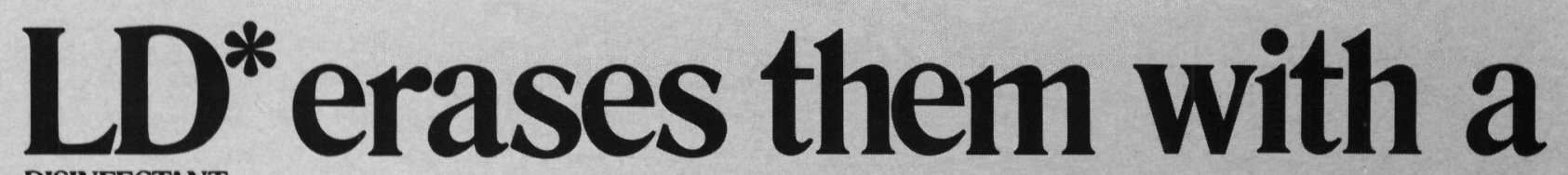

DISINFECTANT

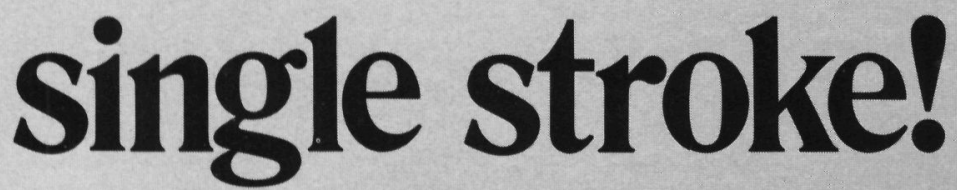

LD $^{m}$ Disinfectant: the only high level disinfectant that:

- kills the broad range of pathogens within 3 minutes;

- doesn't stain or harm soft tissue;

- leaves no toxic residues, and

- flushes safely down the drain!

${ }^{*} \mathrm{LD}^{\mathrm{w}}$ is the Alcide Corporation trade-

mark for its high-level metastabilized chlorous acid/chlorine dioxide

(MECA) disinfectant.

No other class of hard-surface-disinfectant has all these properties.

Not glutaraldehydes. Not phenolics. Not iodophors. Not quats.

In fact, $\mathrm{LD}^{\mathrm{m}}$ Disinfectant is so unique, it's the one single disinfectant to replace all the hard surface disinfectants you now use.

For complete efficacy, safety and pricing information, contact us at the address shown below.

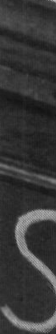
(EPA Reg. No. 45631-15)

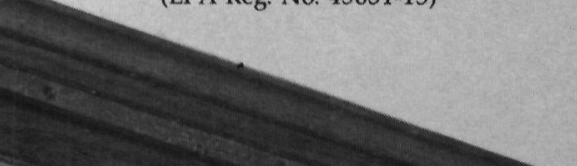

Staphylococcus

seudomonas aureus/A

Herpes Simp Simpl

2/Salmonellach

Poliovirus Type I

Trichophyton

II

nfluenza Vi

Mycobacterium

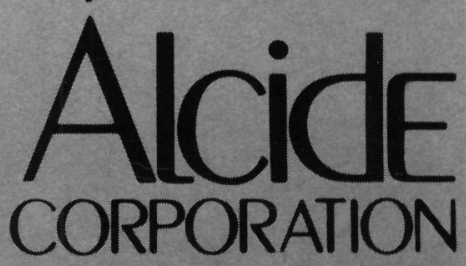

One Willard Road, Norwalk, CT 06851

Phone: 203/847-2555 